\title{
The cost of parallel imaging in functional MRI of the human brain
}

\author{
Henry Lütcke*, Klaus-Dietmar Merboldt, Jens Frahm \\ Biomedizinische NMR Forschungs GmbH am Max-Planck-Institut für Biophysikalische Chemie, 37070 Göttingen, Germany \\ Received 30 June 2005; revised 31 October 2005; accepted 31 October 2005
}

\begin{abstract}
While the advantages of parallel acquisition techniques for echo-planar imaging (EPI) are well documented for studies affected by magnetic field inhomogeneities, this work focuses on the costs in functional MRI of brain regions without artifacts due to susceptibility effects. For a visual stimulation paradigm and relative to conventional EPI ( $2.9 \mathrm{~T}$; TR/TE $=2000 / 36 \mathrm{~ms})$, the use of parallel acquisition at a reduction factor of 2 decreased the mean number of activated voxels by $21 \%$ at $2 \times 2 \times 2-\mathrm{mm}^{3}$ resolution $(n=6)$ and by $15 \%$ at $3 \times 3 \times 3-\mathrm{mm}^{3}$ resolution $(n=6)$. The loss of sensitivity reflects both a decreased signal-to-noise ratio of the native images due to a lower number of contributing gradient echoes and a decreased BOLD MRI sensitivity due to the coverage of a smaller range of TEs.

(C) 2006 Elsevier Inc. All rights reserved.
\end{abstract}

Keywords: Magnetic resonance imaging; Parallel acquisitions; Echo-planar imaging; Functional brain mapping

\section{Introduction}

One of the most promising innovations in the field of magnetic resonance imaging (MRI) is the recent introduction of parallel acquisition techniques [1,2]. At the cost of a somewhat lower signal-to-noise ratio (SNR), pertinent approaches enable substantially faster image acquisition and, for echo-planar imaging (EPI), a reduced sensitivity to magnetic field inhomogeneities and related image artifacts. Parallel acquisition techniques use the distinct spatial sensitivity profiles of individual coils in an array of receive coils to reconstruct undistorted images from individually undersampled data. In practice, two approaches have been developed to achieve this goal. Techniques based on $k$ space derive the missing Fourier lines by combining the weighted sum of the signal from each coil based on individual sensitivity profiles [1]. A few additional lines are usually acquired at the center of $k$ space to improve the reliability of the reconstruction algorithm. In a recent modification of the $k$ space technique [3], these reference lines are acquired before each scan, hence giving rise to the term "generalized autocalibrating partially parallel acquisition" (GRAPPA). Alternatively, image-based methods such as sensitivity encoding (SENSE) attempt to unfold individual aliased

\footnotetext{
* Corresponding author. Tel.: +49 551201 1735; fax: +49 551 2011307.

E-mail address: hluetck@gwdg.de (H. Lütcke).
}

images by solving a system of linear equations based on the known sensitivity profiles of the coils [2]. For both $k$ space- and image-based parallel techniques, the ratio of total $k$-space lines to the sampled $k$-space lines provides a rough estimate of the acceleration of the acquisition process.

Apart from being faster, single-shot gradient-echo sequences such as EPI and spiral imaging may benefit from the fact that the parallel acquisition-related reduction of the echo train length decreases the signal loss and geometric distortions caused by susceptibility differences. This is particularly well documented in diffusion-weighted EPI where no $T_{2}{ }^{*}$ effect is desired [4,5]. For functional MRI (fMRI) of the human brain, the use of parallel acquisition has been reported to increase performance similar to the improvements observed with diffusion-weighted sequences in regions where conventional gradient-echo images suffer from profound susceptibility problems. For example, at 1.5 T, a spiral SENSE technique with an acceleration factor of 2 significantly better recovered orbitofrontal activations associated with a taste paradigm in comparison with spiral imaging without parallel acquisition [6]. In a recent memory study at $3 \mathrm{~T}$, medial temporal lobe activations were observed more reliably when acquired with SENSE-EPI than with EPI as susceptibility-related image distortions were found to be reduced with increasing SENSE acceleration [7].

While there is minimal doubt that parallel fMRI can improve image quality in brain regions affected by magnetic field inhomogeneities, the putative cost of these 
techniques in terms of functional activation has been more controversial. Because of the inherent decrease in SNR associated with GRAPPA or SENSE, any potential decrease of extent or magnitude of the BOLD MRI response to human brain activation needs to be evaluated in areas that are not directly affected by magnetic field inhomogeneities. In contrast to reports of no reduction in activation volume in visual and motor areas [6], two other studies showed consistent $t$ score decreases of 18\% [8] and $7 \%$ [9] in the motor cortex as well as a $20 \%$ decrease of the activation volume due to SENSE-EPI with an acceleration factor of 2 [9].

In view of the increasing number of parallel acquisition applications in functional neuroimaging (see, for example, References $[10,11]$ ), it seems mandatory to assess their overall performance in a slightly more comprehensive manner; that is, in areas of the brain that show limited or no susceptibility effect at all. Thus, the purpose of this work was to address the consequences of GRAPPA in terms of visual functional activation in large parts of the brain not suffering from gradient-echo artifacts. In more detail, the study comprised EPI acquisitions at high $\left(2 \times 2 \times 2 \mathrm{~mm}^{3}\right)$ and low $\left(3 \times 3 \times 3 \mathrm{~mm}^{3}\right)$ spatial resolutions as commonly used in cognitive neuroimaging studies. EPI acquisitions with GRAPPA at an acceleration factor of 2 and at two TEs (36 and $25 \mathrm{~ms}$ ) were compared with conventional EPI at $\mathrm{TE}=36 \mathrm{~ms}$. Functional activation was evaluated with a correlation analysis approach [12] and quantified as the number of activated voxels.

\section{Materials and methods}

Twelve healthy volunteers ( 7 females and 5 males; age range, $18-42$ years; mean age, $27 \pm 6$ years) participated in the study. Informed written consent was obtained from each subject before all examinations were carried out in accordance with institutional guidelines. Three subjects had to be excluded from the analysis because of changes in vigilance during the acquisition of the six protocols (see below) as evidenced by marked reductions or even a complete loss of activations.

\section{1. $M R I$}

All studies were conducted at 2.9 T (Siemens Magnetom Trio, Erlangen, Germany) using an eight-channel receiveonly phased array head coil in combination with a body coil for radio frequency transmission. Each session comprised $T_{1}$-weighted MRI (RF spoiled 3D FLASH; $\mathrm{TR} / \mathrm{TE}=11 / 4.9 \mathrm{~ms}$; flip angle, $15^{\circ}$ ) at $1 \times 1 \times 1-\mathrm{mm}^{3}$ resolution for anatomic referencing.

fMRI was based on a single-shot gradient-echo EPI sequence with frequency-selective fat suppression $\left(\mathrm{TR}=2000 \mathrm{~ms}\right.$; mean $\mathrm{TE}=36$ or $25 \mathrm{~ms}$; flip angle, $\left.70^{\circ}\right)$. For high-resolution scans with a voxel size of $2 \times 2 \times 2 \mathrm{~mm}^{3}$, 16 sections were acquired (no slice gap) using an $84 \times$ 128 acquisition matrix (75\% rectangular FOV of $256 \mathrm{~mm}$;
7/8 partial Fourier encoding in $\mathrm{A}-\mathrm{P}$ direction). Lowresolution scans with a voxel size of $3 \times 3 \times 3 \mathrm{~mm}^{3}$ were based on a $64 \times 64$ matrix (FOV of $192 \mathrm{~mm}$; conventional phase encoding) and comprised 26 sections (no slice gap). In either case, the volume was positioned in an oblique transverse-to-coronal orientation along the calcarine fissure. To approximately match the volume coverage for high- and low-resolution scans, we included only the 11 central sections of the low-resolution scan in the analysis. Motion correction was applied for all BOLD MRI recordings (Siemens Magnetom Trio). EPI acquisitions were performed using GRAPPA, as implemented by the vendor, and a reduction factor of 2 . Twenty-four reference lines were obtained in a single acquisition (external calibration) prior to each functional series. The order of functional recordings with the three sequences studied (conventional EPI as well as EPI with GRAPPA at TEs of 36 and $25 \mathrm{~ms}$ ) was pseudorandomized for each subject as previously described [13].

Functional activation was elicited using a passive visual stimulation task, which compared a flickering $(1 \mathrm{~Hz})$ black-and-white checkerboard with a gray screen. The paradigm was presented as a simple block design where $12 \mathrm{~s}$ of visual stimulation alternated with $18 \mathrm{~s}$ of control. Each experiment started with an 18-s control condition followed by eight experimental cycles. Subjects were instructed to fixate toward a red cross in the center of the screen throughout the experiment. MRI-compatible liquid crystal display goggles (Resonance Technology, Northridge, CA, USA) were used to present visual stimuli $(60-\mathrm{Hz}$ refresh rate; $1024 \times 768$-pixel resolution; visual field, $22.5 \times 30^{\circ}$; virtual eye-to-screen distance, $\sim 120 \mathrm{~cm}$ ). Corrective lenses were applied if necessary.

\subsection{Data analysis}

A correlation analysis of cross-sectional BOLD MRI data was accomplished using in-house software. Activation maps were calculated for a boxcar reference function derived from the task protocol and shifted by $6 \mathrm{~s}$ to account for the delayed hemodynamic response. Significant activations were identified by a statistical evaluation of correlation coefficients following a procedure described previously [12]. First, a histogram of correlation coefficients originating from brain voxels within individual sections is determined. Second, a gaussian curve is fitted to the central portion of the histogram, covering a $50 \%$ range of the observed peak heights extending from the $30 \%$ level to the $80 \%$ level, to estimate the noise distribution of the correlation coefficients. Finally, the distribution of correlation coefficients is rescaled into percentile ranks of the individual noise distribution. Subsequently, pixels are accepted as activated if their correlation coefficients exceed the $99.99 \%$ percentile rank of the noise distribution estimated on an individual basis from the actual measurement. In a second step, neighboring pixels of such activation centers are iteratively added as long as their correlation 
coefficients exceed the $95 \%$ percentile rank of the noise distribution. Importantly, identical thresholds were used for determining significantly activated voxels without and with GRAPPA, thus validating the extent of activation as a true measure of functional contrast. The resulting activation maps were superimposed onto $T_{2}{ }^{*}$-weighted echo-planar images.

The mean number of activated voxels for each of the three sequences was calculated by averaging across subjects. In addition, relative measures of activated voxels for EPI with GRAPPA were calculated by first normalizing individual results to conventional EPI and then averaging across subjects.

Regions of interest (ROIs) were defined in the primary visual cortex to estimate the amount of BOLD MRI signal change associated with stimulus presentation (i.e., the functional contrast). For each of three sections that best covered the sulcus calcarinus, an ROI was placed centrally in the occipital part of the brain comprising $100-150$ voxels $\left(800-1200 \mathrm{~mm}^{3}\right)$ for $2 \times 2 \times 2-\mathrm{mm}^{3}$ resolution and $30-45$ voxels $\left(810-1215 \mathrm{~mm}^{3}\right)$ for $3 \times 3 \times 3-\mathrm{mm}^{3}$ resolution. For individual subjects, the signal intensity time courses were averaged across ROIs and stimulation cycles and normalized to the mean signal strength of the entire time course. Finally, the mean stimulus-induced BOLD MRI signal changes for the six experimental conditions were estimated as the differences between the minimum and the maximum signal intensity of the time courses and averaged across subjects.

\section{Results}

Fig. 1 shows the representative activation maps of two subjects obtained for EPI acquisitions without and with GRAPPA at a reduction factor of 2 for two voxel sizes and TEs. Visual inspection suggests that, relative to conventional EPI, the extent of activation is reduced for GRAPPA versions under all circumstances (i.e., for both voxel sizes and TEs).

The quantitative data summarized in Table 1 confirm a reduction in the mean number of activated voxels for EPI with GRAPPA at identical TEs. If the shorter acquisition period is exploited for a shorter TE of $25 \mathrm{~ms}$, then the extent of activation is decreased even further. When normalized to the number of activated voxels obtained without parallel acquisition, the analysis of the high-resolution data revealed a decrease in activation by $21 \%$ and $29 \%$ for GRAPPA at $\mathrm{TE}=36 \mathrm{~ms}$ and $\mathrm{TE}=25 \mathrm{~ms}$, respectively. With paired $t$ tests, significantly less activation was obtained with GRAPPA at $\mathrm{TE}=25 \mathrm{~ms}$ as compared with conventional $\mathrm{EPI}$ at $\mathrm{TE}=$ $36 \mathrm{~ms}\left[t_{(5)}=2.7 ; P<.05\right]$, whereas the comparison failed to reach significance for GRAPPA at TE $=36 \mathrm{~ms}\left[t_{(5)}=2.2\right.$; $P=.08]$. At a lower resolution, the amount of activation was again clearly reduced by $15 \%$ and by $23 \%$ at $\mathrm{TE}=36 \mathrm{~ms}$ and $\mathrm{TE}=25 \mathrm{~ms}$, respectively, without reaching statistical significance $\left[t_{(5)}=2.1\right.$ and $P=.09$ for $\mathrm{TE}=36 \mathrm{~ms} ; t_{(5)}=2.3$ and $P=.07$ for $\mathrm{TE}=25 \mathrm{~ms}]$.

To characterize the extent of activation further, as well as to optimize the statistical power of the comparison, we

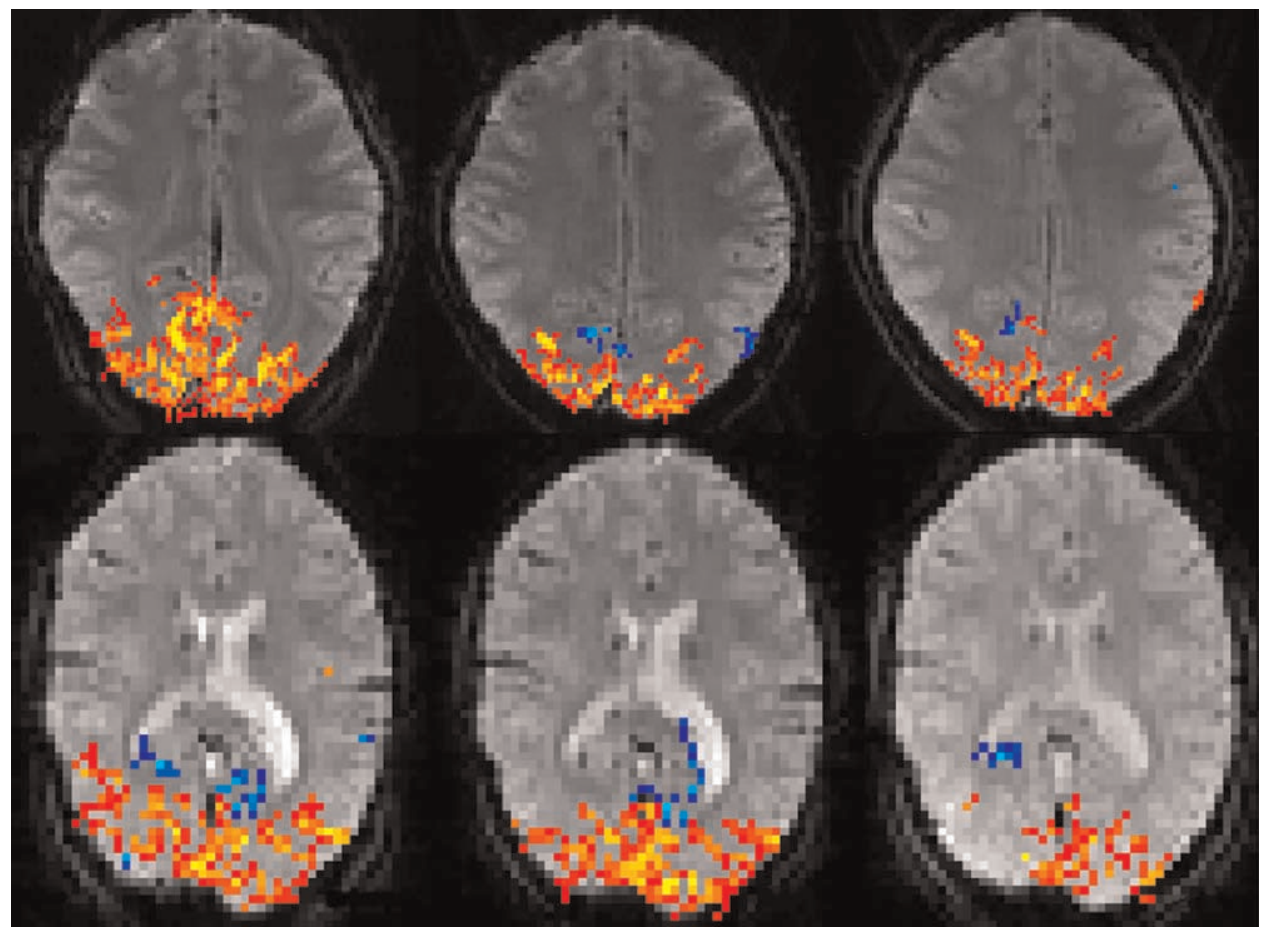

Fig. 1. Representative activation maps obtained for (top) $2 \times 2 \times 2-\mathrm{mm}^{3}$ resolution and (bottom) $3 \times 3 \times 3-\mathrm{mm}^{3}$ resolution (two subjects). The maps represent acquisitions with (left) conventional EPI at TE $=36 \mathrm{~ms}$, (middle) EPI with GRAPPA at a reduction factor of 2 and TE $=36 \mathrm{~ms}$ and (right) EPI with GRAPPA at a reduction factor of 2 and $\mathrm{TE}=25 \mathrm{~ms}$. Red-yellow= positive correlations; blue=negative correlations. 
Table 1

Number of activated pixels and BOLD MRI signal change without and with GRAPPA at a reduction factor of 2

\begin{tabular}{lccc}
\hline EPI & $\begin{array}{l}\text { Normal } \\
(36 \mathrm{~ms})\end{array}$ & $\begin{array}{l}\text { GRAPPA } \\
(36 \mathrm{~ms})\end{array}$ & $\begin{array}{l}\text { GRAPPA } \\
(25 \mathrm{~ms})\end{array}$ \\
\hline $\begin{array}{l}2 \times 2 \times 2 \mathrm{~mm}^{3} \\
\text { TE range }(\mathrm{ms})\end{array}$ & $8-74$ & $22-55$ & $11-44$ \\
Activated pixels & $4482 \pm 1405$ & $3313 \pm 392$ & $3052 \pm 594$ \\
Normalized & 1.00 & $0.79 \pm 0.21^{*}$ & $0.71 \pm 0.15^{* *}$ \\
Signal change $(\%)$ & $2.56 \pm 0.83$ & $2.48 \pm 0.86$ & $1.77 \pm 0.56^{* *}$ \\
& & & \\
$3 \times 3 \times 3 \mathrm{~mm}^{3}$ & & & $12.5-37.5$ \\
TE range (ms) & $10-61$ & $1385 \pm 444$ & $1208 \pm 331$ \\
Activated pixels & $1599 \pm 336$ & $0.85 \pm 0.18^{*}$ & $0.77 \pm 0.21^{*}$ \\
Normalized & 1.00 & $2.69 \pm 1.03$ & $1.96 \pm 0.80^{* *}$ \\
Signal change $(\%)$ & $2.77 \pm 1.07$ & & \\
\hline
\end{tabular}

Values are given as mean \pm S.D. $\left(n=6\right.$ for $8-\mathrm{mm}^{3}$ voxels and $n=6$ for $27-\mathrm{mm}^{3}$ voxels). Normalized values are relative to the number of activated pixels obtained for EPI without PPA. Mean BOLD MRI signal change is given in percentage of mean signal strength in standardized ROIs of the primary visual cortex.

* $P<.1$ (paired $t$ test).

** $P<.05$ (paired $t$ test).

combined low- and high-resolution data in a $3 \times 2$ (Sequence $\times$ Resolution) mixed analysis of variance. This procedure demonstrated a highly significant decrease in the extent of activation for GRAPPA-supported acquisitions $\left[F_{(2,20)}=\right.$ $7.5 ; P<.01]$. The interaction between the factors Sequence and Resolution was nonsignificant $\left[F_{(2,20)}=2.8 ; P=\mathrm{NS}\right]$, which demonstrates that the reduction in activation volume due to parallel imaging is similar for high- and lowresolution acquisitions. Planned comparisons of activation volume revealed a significantly reduced number of activated voxels both for GRAPPA at TE $=36 \mathrm{~ms}\left[t_{(11)}=2.3 ; P<.05\right]$ and for that at TE $=25 \mathrm{~ms}\left[t_{(11)}=3.0 ; P<.05\right]$ as compared with conventional acquisition (corrected for multiple comparisons using the procedure set forth by Holm [14]).

With a standardized set of ROIs in the primary visual cortex, the BOLD MRI signal change was significantly reduced for EPI with GRAPPA at TE $=25 \mathrm{~ms}$ as compared with both conventional EPI and EPI with GRAPPA at $\mathrm{TE}=36 \mathrm{~ms}$ for high resolution $\left[t_{(5)}=4.4, P<.05\right.$, and $t_{(5)}=3.9, P<.05$, respectively $]$ and low resolution $\left[t_{(5)}=\right.$ $6.1, P<.05$, and $t_{(5)}=4.6, P<.05$, respectively].

\section{Discussion}

The present study revealed a substantial reduction in functional activation (as assessed by the number of activated voxels) when using EPI in conjunction with GRAPPA at a reduction factor of 2 . The finding applies to large parts of the occipital and parietal cortices (i.e., regions that are only mildly or not at all affected by susceptibility problems and related EPI distortions). Reduction of the activation volume due to GRAPPA alone amounted to $15 \%$ and $21 \%$ for lowand high-resolution sequences, respectively, whereas a concurrent decrease of the TE diminished the extent of activation even more.
The proportion of the total variance in the extent of activation explained by parallel imaging may be calculated by dividing the sum of squares of the effect by the total sum of squares (Effect+Interaction+Error Term):

$\eta^{2}=\frac{\mathrm{SS}_{\text {effect }}}{\mathrm{SS}_{\text {total }}}$

With $\eta^{2}$ as a measure of the effect size, parallel imaging accounted for $37 \%$ of the total variability in the extent of activation. Considering the large between-subject variability typical for fMRI studies, the effect of GRAPPA on the activation volume must be considered as substantial.

The observed loss of overall BOLD sensitivity with GRAPPA may be attributed to at least two independent factors. First, because the number of sampled $k$-space lines is reduced for GRAPPA, the SNR is also reduced. Second, an independent source of BOLD contrast attenuation becomes evident when considering the range of TEs covered by EPI while scanning $k$ space (compare Table 1 ). GRAPPA alone caused a reduction of the TE range by approximately $50 \%$, whereas a mean TE of only $25 \mathrm{~ms}$ further decreased the range of absolute TE values. The mean TE should be approximately equal to tissue $T_{2}{ }^{*}$, which at $3 \mathrm{~T}$ is approximately $40-50 \mathrm{~ms}$, to achieve optimal BOLD MRI sensitivity. Table 1 shows that this range is covered sufficiently well by EPI without and even with parallel acquisition as long as the mean TE is kept at $36 \mathrm{~ms}$. Accordingly, the achievable BOLD MRI signal change was very similar for both conditions. In contrast, a combination of GRAPPA with a TE of only $25 \mathrm{~ms}$ moves the upper limit of the acquired TE range near the TE value for optimal $T_{2}{ }^{*}$ weighting. Consequently, a large part of $k$ space, and particularly its center, is acquired with TEs that are far too low to result in effective $T_{2}{ }^{*}$ weighting and corresponding BOLD MRI sensitivity. This observation is supported by the approximately $30 \%$ decrease of the BOLD MRI signal change at $\mathrm{TE}=25 \mathrm{~ms}$ for both high and low spatial resolutions.

Our results were obtained using a $k$ space-based parallel acquisition technique and correlation analysis of functional time series, yet they are in broad agreement with previous results by others, one of which, for example, reported a $20 \%$ reduction in the extent of motor cortex activation for SENSE-EPI [9]. Moreover, a recent comparison of EPI with SENSE-EPI obtained larger activation clusters in the inferior frontal gyrus, which hardly suffers from susceptibility artifacts, when the acquisition was performed without parallel acquisition, whereas improvements with SENSE were seen in critical regions such as the anterior hippocampus, amygdala and fusiform gyrus [7].

\section{Conclusions}

The present study demonstrates that fMRI of human brain activation may suffer from the use of parallel acquisition techniques. In comparison with conventional 
EPI, the combination of EPI with GRAPPA at a reduction factor of 2 incurs a substantial cost on BOLD MRI sensitivity in brain areas not or only mildly affected by magnetic field inhomogeneities. The $20 \%$ reduction of the extent of activation observed here must be considered as a substantial loss, especially in view of cognitive neuroimaging studies where activated areas tend to be small. Investigators planning GRAPPA-supported fMRI studies certainly are knowledgeable about the benefits in terms of reduced susceptibility artifacts and scan time, but they should also be aware of the associated cost due to a lower SNR and partial loss of $T_{2} *$ weighting. It may be advisable to invest the saved time into signal averaging for improved SNR while retaining the parallel acquisition advantages with respect to susceptibility artifacts to achieve a reasonable compromise between enhanced speed, improved image quality and reduced BOLD MRI sensitivity.

\section{References}

[1] Sodickson DK, Manning WJ. Simultaneous acquisition of spatial harmonics (SMASH): fast imaging with radiofrequency coil arrays. Magn Reson Med 1997;38:591-603.

[2] Pruessmann KP, Weiger M, Scheidegger MB, Boesiger P. SENSE: sensitivity encoding for fast MRI. Magn Reson Med 1999;42: $952-62$.

[3] Griswold MA, Jakob PM, Heidemann RM, Nittka M, Jellus V, Wang J, et al. Generalized autocalibrating partially parallel acquisitions (GRAPPA). Magn Reson Med 2002;47:1202-10.
[4] Bammer R, Keeling SL, Augustin M, Pruessmann KP, Wolf R, Stollberger $\mathrm{R}$, et al. Improved diffusion-weighted single-shot echoplanar imaging (EPI) in stroke using sensitivity encoding (SENSE). Magn Reson Med 2001;46:548-54.

[5] Jaermann T, Crelier G, Pruessmann KP, Golay X, Netsch T, van Muiswinkel AM, et al. SENSE-DTI at 3 T. Magn Reson Med 2004; 51:230-6.

[6] Weiger M, Pruessmann KP, Osterbauer R, Bornert P, Boesiger P, Jezzard P. Sensitivity-encoded single-shot spiral imaging for reduced susceptibility artifacts in BOLD fMRI. Magn Reson Med 2002; 48:860-6.

[7] Schmidt CF, Degonda N, Luechinger R, Henke K, Boesiger P. Sensitivity-encoded (SENSE) echo planar fMRI at $3 \mathrm{~T}$ in the medial temporal lobe. Neuroimage 2005;25:625-41.

[8] de Zwart JA, van Gelderen P, Kellman P, Duyn JH. Application of sensitivity-encoded echo-planar imaging for blood oxygen leveldependent functional brain imaging. Magn Reson Med 2002;48: $1011-20$.

[9] Preibisch C, Pilatus U, Bunke J, Hoogenraad F, Zanella F, Lanfermann H. Functional MRI using sensitivity-encoded echo planar imaging (SENSE-EPI). Neuroimage 2003;19:412-21.

[10] Kirwan CB, Stark CE. Medial temporal lobe activation during encoding and retrieval of novel face-name pairs. Hippocampus 2004; 14:919-30.

[11] Okado Y, Stark CE. Neural activity during encoding predicts false memories created by misinformation. Learn Mem 2005;12:3-11.

[12] Baudewig J, Dechent P, Merboldt KD, Frahm J. Thresholding in correlation analyses of magnetic resonance functional neuroimaging. Magn Reson Imaging 2003;21:1121-30.

[13] Williams EJ. Experimental designs balanced for the estimation of residual effects of treatments. Aust J Sci Res Ser A 1949;2:149-68.

[14] Holm S. A simple sequentially rejective multiple test procedure. Scand J Stat 1979;6:65-70. 